Corresponding author: Ali Khalighifar, Biodiversity Institute, University of Kansas, 1345 Jayhawk Blvd, Lawrence, KS 66045, +1 (785) 925-8041, a.khalighifar@ku.edu

\title{
Deep learning algorithms improve automated identification of Chagas disease vectors
}

\section{Ali Khalighifar ${ }^{1}$, Ed Komp ${ }^{2}$, Janine M. Ramsey ${ }^{3}$, Rodrigo Gurgel-Gonçalves ${ }^{4}$, and A. Townsend Peterson $^{1}$}

${ }^{1}$ Biodiversity Institute and Department of Ecology and Evolutionary Biology, University of Kansas, Lawrence, KS, USA

${ }^{2}$ Information and Telecommunication Technology Center, University of Kansas, Lawrence, KS, USA

${ }^{3}$ Centro Regional de Investigación en Salud Pública, Instituto Nacional de Salud Publica, Tapachula, Chiapas, Mexico

${ }^{4}$ Faculty of Medicine, Universidade de Brasília, Brasilia, DF, Brazil

\begin{abstract}
Vector-borne Chagas disease is endemic to the Americas and imposes significant economic and social burdens on public health. In a previous contribution, we presented an automated identification system that was able to identify 12 Mexican and 39 Brazilian triatomine (Hemiptera: Reduviidae) species from digital images. To explore the same data more deeply using machine-learning approaches, hoping for improvements in classification, we employed TensorFlow, an open-source software platform for a deep-learning algorithm. We trained the algorithm based on 405 images for Mexican triatomine species
\end{abstract}


and 1584 images for Brazilian triatomine species. Our system achieved 83.0 and $86.7 \%$ correct identification rates across all Mexican and Brazilian species, respectively, an impressive improvement over comparable rates from statistical classifiers ( 80.3 and $83.9 \%$, respectively). Incorporating distributional information to reduce numbers of species in analyses improved identification rates to $95.8 \%$ for Mexican species and $98.9 \%$ for Brazilian species. Given the "taxonomic impediment" and difficulties in providing entomological expertise necessary to control such diseases, automating the identification process offers a potential partial solution to crucial challenges.

Key words: Chagas disease, TensorFlow, Deep learning, Triatominae, Automated species identification

\section{Introduction}

Vector-borne diseases, such as malaria, Zika, dengue fever, Chagas disease, and chikungunya, impose enormous economic and social burdens on public health (Sinkins and Gould 2006), and rank among the most serious threats to human health (Nauen 2007). An important element in predicting risk of such diseases, and developing strategies to control them, is detailed surveillance of arthropod vector species (Mukundarajan et al. 2017). Identifying vectors can be challenging, requiring significant training and increasingly uncommon taxonomic expertise (Drew 2011), a phenomenon termed the "taxonomic impediment" (de Carvalho et al. 2007). As a result, providing broad regions with necessary arthropod vector identification expertise by traditional means is often not feasible given political boundaries, logistical obstacles, and variability of country-specific human health resources (Schofield et al. 2006). One promising potential solution involves developing automated species identification systems that can aid in meeting these important challenges. 
Chagas disease occurs in most countries in the Americas, causing serious cardiac morbidity and mortality among infected individuals over years or decades if untreated (Coura and Viñas 2010). The disease is caused by the unicellular parasitic protozoan, Trypanosoma cruzi (Trypanosomatida: Trypanosomatidae), transmitted mainly through feces of blood-sucking true bugs belonging to the hemipteran subfamily Triatominae (Hemiptera: Reduviidae) (Coura and Viñas 2010). Estimates suggest that 8 million people exhibit symptoms of the disease, and that $>100$ million people are at risk of infection worldwide (Coura and Viñas 2010, WHO 2018). Given failings in many public health systems across the Americas, epidemiological surveillance for Chagas disease is either minimal (e.g., in Mexico) or incomplete (e.g., in Brazil), and diagnosis and treatment are often too late and therefore less effective (Martins-Melo et al. 2012, Ramsey et al. 2014).

Many efforts have tested diverse inferential approaches in the challenge of identifying insects accurately. Recently, a new set of machine-learning classifiers, called deep neural networks (DNNs), have been designed; they are similar to artificial neural networks (ANNs; Dayhoff and DeLeo 2001), but with multiple hidden layers between the input and output layers, instead of having only three layers total (i.e., input, one hidden layer, and output). The high capability of DNNs has resulted in widespread use of these techniques in other such inference challenges (Schmidhuber 2015). DNNs have outperformed traditional methods (e.g., linear discriminant analyses) in various classification tasks (Schmidhuber 2015). Here, we used TensorFlow (Abadi et al. 2016), an open-source software platform, representing the most recent addition to the deep-learning toolbox (Rampasek and Goldenberg 2016; Google Brain Team; https://research.google.com/teams/brain/), to explore the challenge of automated Chagas disease vector identification.

The purpose of this study was to test whether deep-learning techniques can improve abilities to identify Chagas disease vectors of Mexico and Brazil successfully, using a collection of digital images of triatomines from our previous work (Gurgel-Gonçalves et al. 2017). To address this question, we designed 
an automated, DNN-based species identification system for 12 Mexican and 38 Brazilian species, which we tested using images withheld from the calibration process. This study thereby lays a foundation for many automated identification capabilities in medical entomology, and more broadly in biodiversity science.

\section{Materials and Methods}

We tested whether state-of-the-art, deep-learning techniques (TensorFlow; Abadi et al. 2016) can successfully identify digital images of 12 Mexican triatomine species and 38 Brazilian triatomine species. We implemented four steps: (1) adapt and configure TensorFlow to the needs of this analysis; (2) create different sets of images, based on different levels of automated pre-processing, from available images, as inputs; (3) apply and test TensorFlow under diverse circumstances; and (4) use secondary information (i.e., distributional information) to reduce numbers of species in our identification exercises. We implemented all analyses in Python (version 2.7, available at http://www.python.org/). These steps are described in greater detail below.

Previously (Gurgel-Gonçalves et al. 2017), we presented work on automated identification of triatomine species from Mexico (12 species) and Brazil (39 species). That work involved full automation of processing of images before analysis into a set of landmarks and distances among them, and then analysis using traditional statistical classifiers, such as linear discriminant analysis (Fisher 1936) and artificial neural networks (Dayhoff and DeLeo 2001). Identifying the Mexican species was a particular challenge to our classifiers, and of course we were interested in improving identification rates for Brazilian species also. Therefore, we decided to use more advanced classifiers for both Mexican and Brazilian triatomines. To be able to compare our results, we used the same species and same images for this study (Appendix 1, Appendix 2); however, given small numbers of images available for Eratyrus mucronatus (Stål) (Hemiptera: Reduviidae) ( $n=11)$, we removed this species from the Brazilian pool. 


\section{Photographs as input}

In our previous study, we had observed that most triatomine images then available were of poor quality, such that the resulting identifications were not reliable. Consequently, project personnel designed a photo apparatus that would permit taking consistent, high quality, repeatable photographs of triatomines at low cost (Gurgel-Gonçalves et al. 2017). This apparatus allows users to employ iPods or cell phones for taking photographs. Team members photographed triatomine species from various institutions across Mexico (Centro Regional de Investigación en Salud, Instituto Nacional de Salud Pública México; Laboratorio Estatal de Salud Pública de Guanajuato; Universidad Autónoma Benito Juárez, Oaxaca; Universidad Autónoma de Nuevo León, Monterrey), and Brazil (Universidade de Brasília, FIOCRUZ Bahia, FIOCRUZ Rio de Janeiro, FIOCRUZ Minas Gerais, Universidade Estadual Paulista), using the 5th and 6th generations of iPods. Detailed information about the apparatus and protocols for capturing images is provided in Gurgel-Gonçalves et al. (2017).

The photographs that we used as our "reference library" for our identifications are of high-quality, and are openly available at http://dx.doi.org/10.5061/dryad.br14k; they are described in great detail by Gurgel-Gonçalves et al. (2017). These photographs are consistent in orientation, background color, pixel resolution, and quality, which made automation of their processing feasible. Subsequent steps were automated (Gurgel-Gonçalves et al. 2017), including removing (digitally) the background from the images, and identifying the body edge, yielding two sets of images (raw photographs, and images cleaned to remove background) to understand how deep-learning would perform with different quality levels of images as input (Fig. 1). The processing step was automated fully and the program code is openly available in the appendices of our previous paper (Dryad Digital Repository: http://dx.doi.org/10.5061/dryad.br14k; Gurgel-Gonçalves et al. 2017). 

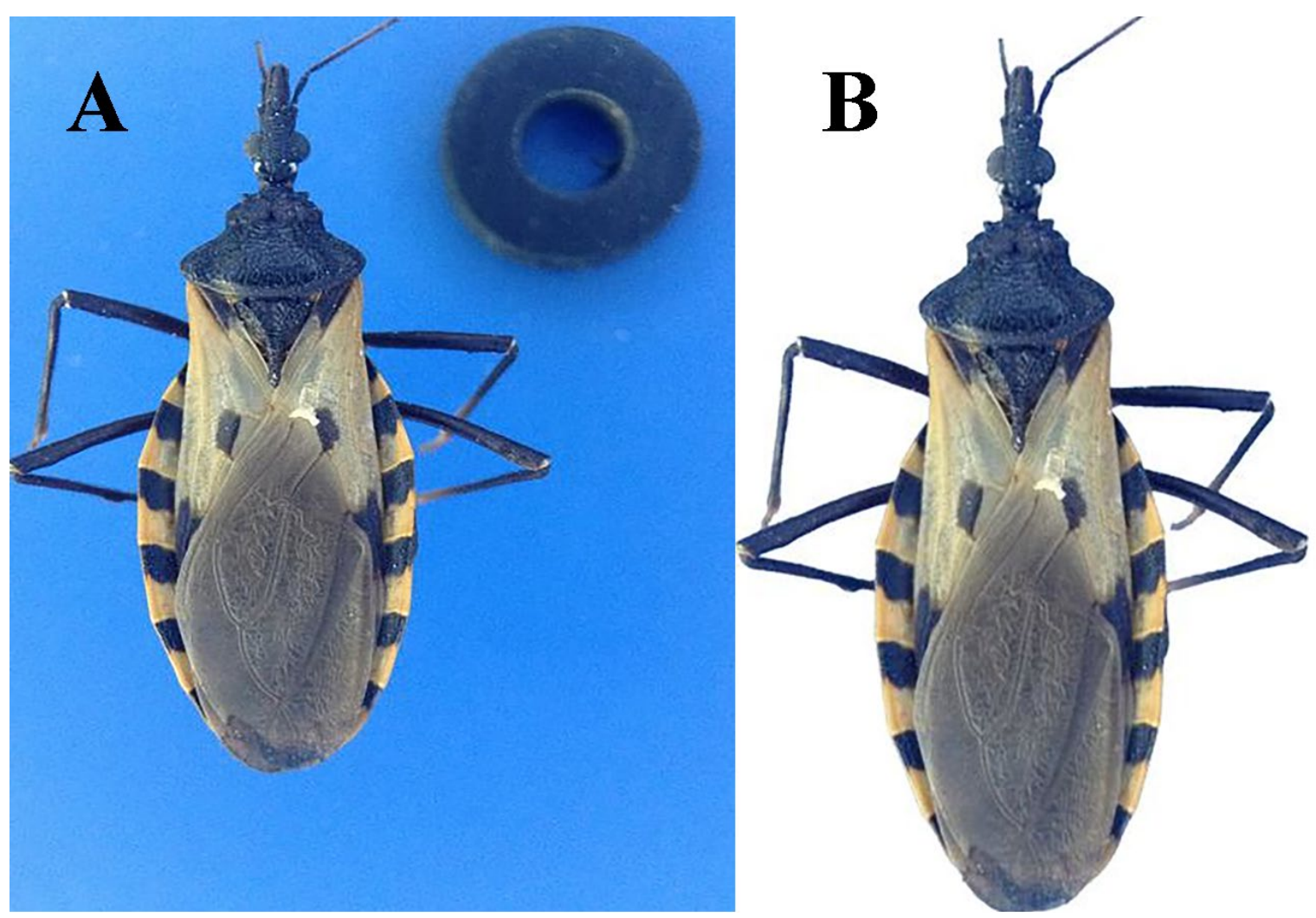

Fig. 1. An example image of an individual of Triatoma dimidiata $\mathrm{Hg} 1$. (A) raw image, (B) final image with background removed digitally.

\section{TensorFlow}

TensorFlow is an open-source software platform (Abadi et al. 2016) that supports deep-learning research and applications, having been released by researchers at Google (Google Brain Team; https://research.google.com/teams/brain/). We adapted and configured TensorFlow for application and use in our project via Python. We adjusted two parameters from their default values: validation percentage and number of training steps. Although the default setting for validation percentage was $10 \%$, given small numbers of images available for some species, we had to increase this number to $16 \%$. For number of training steps, although the default value was 4000 steps, we explored different numbers and compared the results to find the optimum balance between computing time and classification efficiency. 
To evaluate the classifier, it is necessary to set some images aside from classifier development entirely. Given the limited numbers of photographs available for some of the species, we used a leaveone-out cross-validation approach for evaluation. That is to say, as a trade-off between maximizing numbers of samples for training the classifier and optimizing computing time, in each round, we left out $n$ images, or one image from among the $n$ species in that country, with which to evaluate the model.

\section{Identifying faunal subsets based on distributional information}

Generally, classifier accuracy decreases as the number of classes that must be distinguished increases. In the preliminary work, triatomine species were immediately separated into two independent groups; those occurring in Mexico and those in Brazil. This separation is natural since almost no triatomine species occur naturally in both countries, exception for Panstrongylus rufotuberculatus (Champion) (Hemiptera: Reduviidae). Independent classifiers were created for the two groups. Within these two groups, we applied the same technique on a finer scale, to determine if using a specialized classifier that discriminates only among species known to be present near the location at which a sample is taken will improve the ability to correctly classify triatomine samples. We used the same binary maps as in our previous study (Gurgel-Gonçalves et al. 2017), summarizing potential geographic distributions for each of the 12 Mexican and 38 Brazilian species. Ecological niche modeling techniques and data sources are described in detail in Gurgel-Gonçalves et al. (2012), Ramsey et al. (2015), and Gurgel-Gonçalves et al. (2017). Hence, in Python 2.7, we created 399 and 2019 local fauna sets for the Mexican and Brazilian triatomine species, respectively, using the distributional information to create subsets of species that cooccur at the same sites. We trained and tested the classifier employing samples restricted to those local faunas. The range of numbers of species in local fauna subsets was 2-11 for Mexican species and 2-12 for Brazilian species. 


\section{Results}

As a preliminary step, we tested identification success for raw versus cleaned images for Mexican species, to see whether TensorFlow shows reduced correct identification rates using raw images. The identification rate for raw images was almost the same as that obtained using the cleaned images (i.e., 82.9\% overall identification rate for raw images, versus $83.0 \%$ for cleaned images); since our goal was to compare the results of TensorFlow with those deriving from the statistical classifiers used in our previous study (Gurgel-Gonçalves et al. 2017), for all succeeding analyses in this study, we elected to use cleaned images, only noting the potential for simpler processing in future work.

We calibrated our models for Mexican and Brazilian species using different numbers of training steps, and considered two factors to find optima for training TensorFlow (Fig. 2): (1) correct identification rate, which improved up to a certain point, but then reached a plateau, beyond which we saw no significant improvement, and (2) processing time (in hours), which showed a positive linear relationship with number of training steps. For the Mexican dataset, with 12 species, 2000 emerged as an optimum number of training steps, which resulted in an $83.0 \%$ overall identification rate (Fig. 2); the largest gap between the processing time trace and identification rate occurred following this number of steps. Above this number of training steps, subtle improvements in identification rate were possible (up to $83.1 \%$ ), but processing time was significantly longer (7 hours instead of 3.5 hours). Based on the same logic, we chose 12,000 training steps as an optimum number for Brazilian species, at which we achieved an $86.7 \%$ overall identification rate (Fig. 2). The Brazilian dataset had a plateau that was more obvious: overall identification rate barely reached a maximum $87.0 \%$, yet processing time increased from 30 to 80 hours. It should be noted that the amount of time discussed above is only for training TensorFlow over all images in the reference library with multiple replicate analyses; for identification of a single image, it only takes a few seconds. 

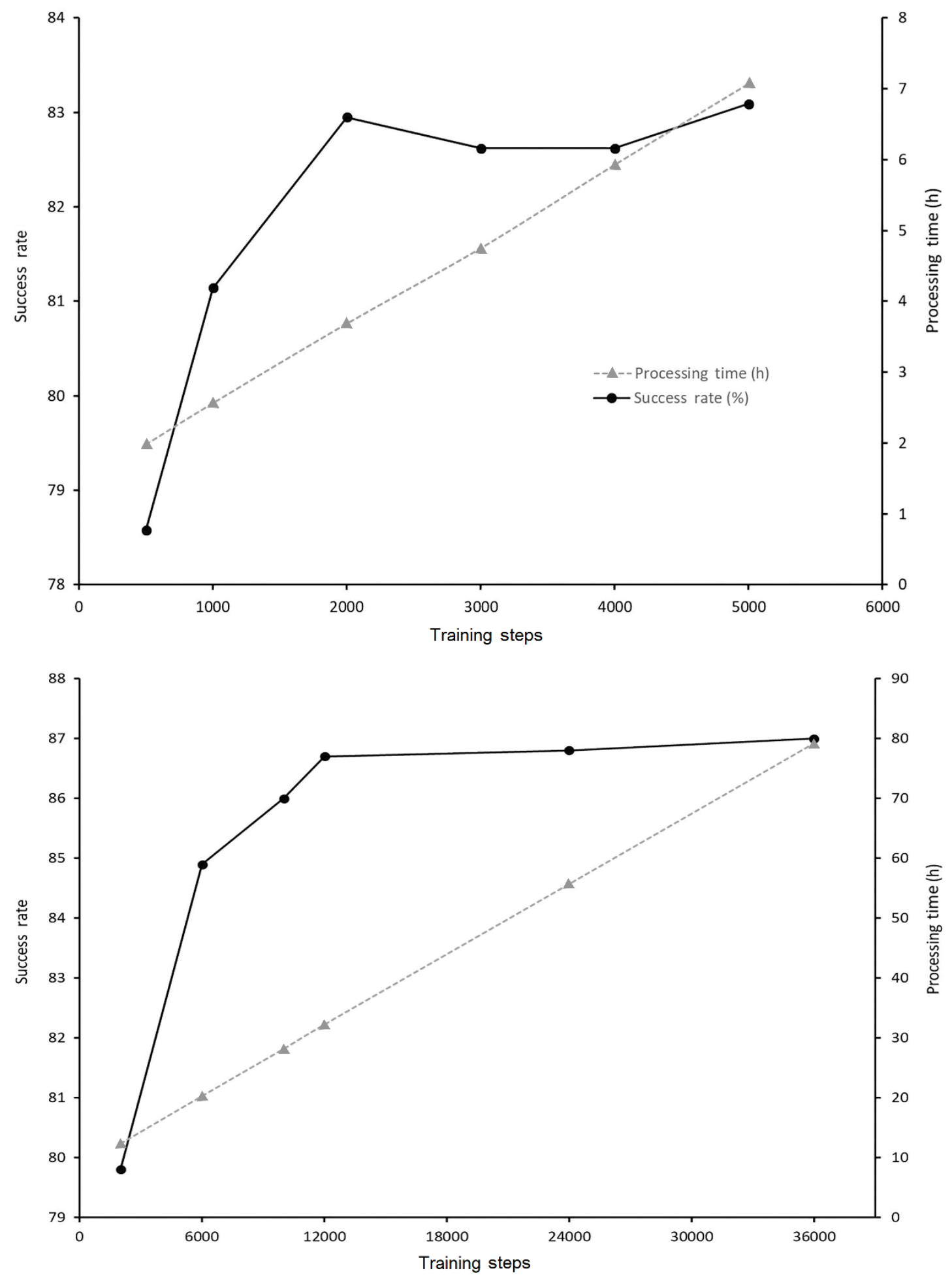

Fig. 2. Effects of different numbers of training steps on identification rate (\%) and processing time (h) using TensorFlow for Mexican (top) and Brazilian (bottom) triatomine species. 
We plotted and compared the results of TensorFlow with those from our previous study (GurgelGonçalves et al. 2017), at the species level (Fig. 3). We applied a paired $t$-test on outcomes, which produced $p$-values of 0.028 and 0.025 for Mexican and Brazilian triatomines, respectively; given that the $p$-values were $<0.05$, we concluded that the DNN-based results are statistically significantly better than our previous results. For Mexican species, we noted improvement in identification rate for 9 out of 12 species using TensorFlow (Fig. 3). Although T. mazzottii Usinger correct identification rates declined from 77.3\% to $63.6 \%$ using deep learning, we noted significant improvement for the $T$. dimidiata (Latreille) complex (T. dimidiata $\mathrm{Hg} 1$ increased from $70.5 \%$ to $84.1 \%$, $T$. dimidiata $\mathrm{Hg} 2$ from $76.7 \%$ to $86.7 \%$, and $T$. dimidiata $\mathrm{Hg} 3$ from $82.5 \%$ to $87.5 \%$ ), T. mexicana (Herrich-Schaeffer) from $80.0 \%$ to $97.8 \%$, and T. barberi from $72.4 \%$ to $80.0 \%$. Most notably, we saw improvements for T. nitida Usinger and T. phyllosoma (Burmeister, 1835), which were very challenging for our previous classifiers, from $46.7 \%$ to $73.3 \%$ and $46.6 \%$ to $69.0 \%$, respectively. For Brazilian species, identification rate improved for 23 out of 38 species, including 6 with major improvements: T. vandae Carcavallo, Jurberg, Rocha, Galvão, Noireau \& Lent from $69.0 \%$ to $79.3 \%$, T. sordida (Stål, 1859) from $81.2 \%$ to $92.7 \%$, T. rubrovaria (Blanchard, 1843) from 59.3\% to $79.6 \%$, T. melanica Neiva \& Lent from $79.3 \%$ to $89.3 \%$, T. circummaculata (Stål) from $85.7 \%$ to $95.5 \%$, and T. brasiliensis Neiva from $76.6 \%$ to $92.2 \%$; identification rates for 5 out of 38 species stayed the same (Fig. 3). A highlight was that $T$. rubrovaria had shown the weakest classification success with statistical classifiers, but with deep learning improved dramatically. However, 3 species showed major declines: $R$. milesi Carcavallo, Rocha, Galvão \& Jurberg (Hemiptera: Reduviidae) from $89.2 \%$ to $74.4 \%$, $T$. delpontei Romana \& Abalos from $86.7 \%$ to $74.2 \%$, and T. maculata (Erichson) from $89.7 \%$ to $77.5 \%$, compared with the results of the statistical classifiers. The confusion matrices for both Mexican and Brazilian species, summarizing numbers of images for each species identified correctly or misidentified as another species, are presented in the supplementary data of this paper. 
$\square$ TensorFlow

a Statistical classifiers

\section{Mexican species}
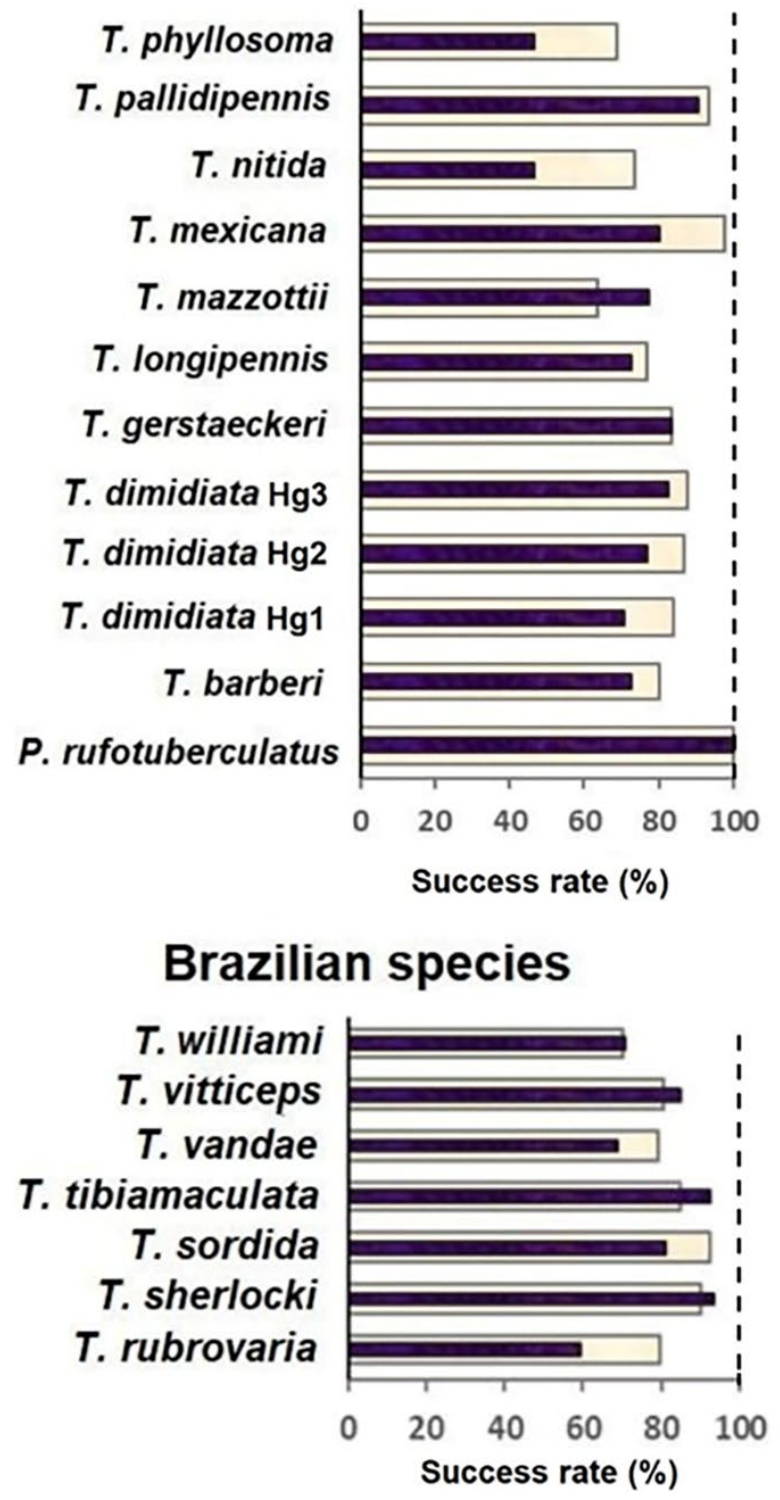

\section{Brazilian species}

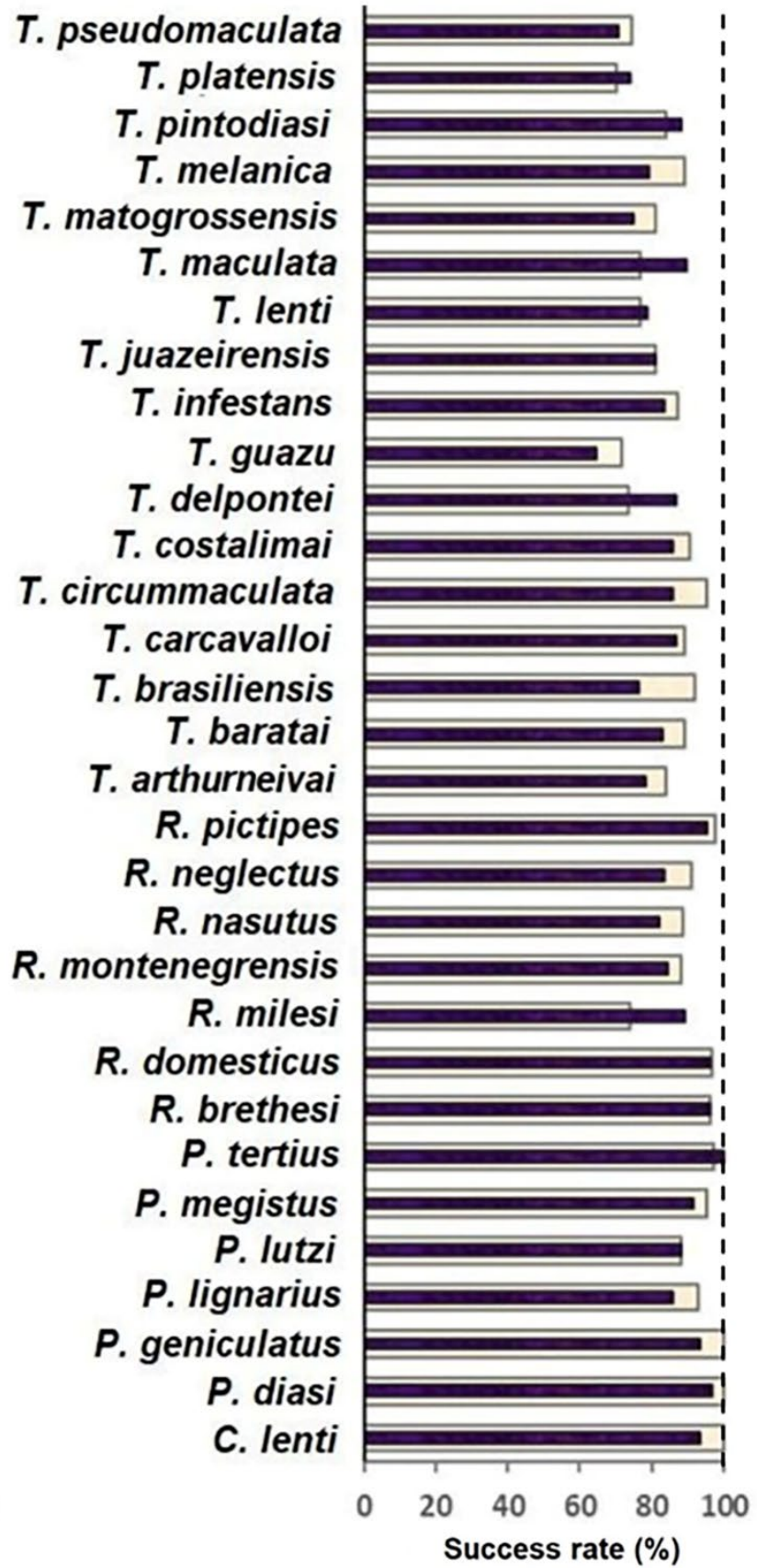

Fig. 3. Comparison of identification rates (\%) between TensorFlow and statistical classifiers (GurgelGonçalves et al. 2017) at species level for Mexican and Brazilian triatomines. 


\section{Faunal Subsets}

Because classifier accuracy improves when fewer classes are compared, to reduce numbers of species being compared, we used geographic information for the distributions of Mexican and Brazilian triatomines to refine identification efforts, testing 399 and 2019 faunal subsets for the two countries, respectively. For Mexican species, faunal subsets ranged 2-11 species (Fig. 4). TensorFlow was able to increase the overall identification rate for comparing two species up to $95.8 \%$; average identification rates for most local faunas (25 out of 31 ) were in the range of $95-100 \%$. As expected, increasing the number of species compared, the overall identification rate declined gradually, and leveled off at around $83.0 \%$ for 8 to 11 Mexican species. For Brazilian species, the overall identification rate for comparing two species was $98.9 \%$ (Fig. 4). Adding more species to the Brazilian pool, the overall identification rate decreased to a plateau of about $94.0 \%$ with 11 species. For 1853 out of 2019 Brazilian local fauna subsets, TensorFlow's identification performance was $95 \%$ or better. 


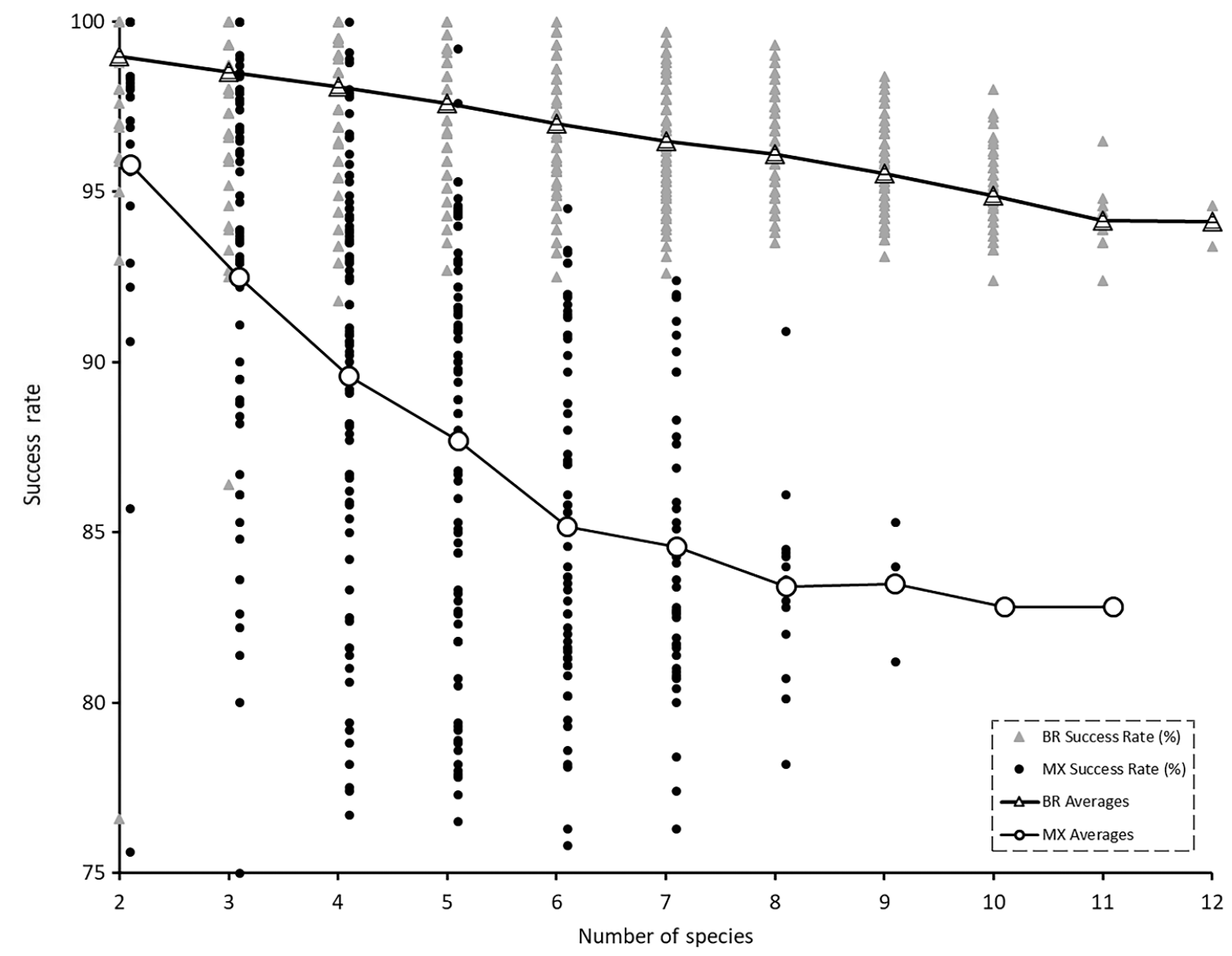

Fig. 4. Summary of identification rates (\%) for various combinations of species: 2-11 different species combinations for Mexican faunas with 399 faunal subsets, and 2-12 species combinations for Brazilian faunas with 2019 faunal subsets, using the distributional information. Gray triangles represent average identification rates for each Brazilian local fauna, and large black triangles show the overall average of identification rate for each fauna size. Black dots represent average identification rates for each Mexican local fauna, and large blank circles show the overall average of identification rate for each fauna size. 


\section{Discussion}

Chagas disease causes serious cardiac morbidity and mortality among infected individuals over the course of years or decades (Coura and Viñas 2010), yet epidemiological surveillance, diagnosis, and treatment are often late or inefficient owing to shortage of expertise and failings in many public health systems (Martins-Melo et al. 2012, Ramsey et al. 2014). Arriving at such diagnoses depends rather crucially on vector awareness and correct identification, which is the focus of our work. To that end, we have explored deep neural networks (Schmidhuber 2015) to automate several key inferential tasks. We successfully identified both Mexican and Brazilian triatomine species with considerable improvement in overall identification rates, compared to our previous results (Gurgel-Gonçalves et al. 2017). Incorporating distributional information for species allowed us to reduce local fauna sizes in analyses, making identification easier, which increased overall identification rates for local faunas still more.

A crucial advantage of using TensorFlow was eliminating the need for pre-processing images for our identification system. That is, we compared TensorFlow classification performance based on the cleaned and raw images for the Mexican species, which were the most challenging to our statistical classifiers (Gurgel-Gonçalves et al. 2017). TensorFlow achieved almost the same overall identification rate using the raw images, which opens important future possibilities, such as developing identification based on photos taken by local residents with mobile phone cameras. Including geographic information to create local faunas or faunal subsets (Gurgel-Gonçalves et al. 2012, Ramsey et al. 2015) significantly improved our results, reaching an average accuracy of $95.8 \%$ for Mexican triatomines and $98.9 \%$ for Brazilian triatomines. Another advantage of TensorFlow is that it is open source software: TensorFlow's flexible architecture allows easy adaptation and deployment of the software on different platforms including desktops, clusters of servers, edge devices, and mobile phones (https://www.tensorflow.org/). 


\section{Deep neural networks}

Applying TensorFlow to our dataset presented some challenges, a major one was the number of images available for each species. To the best of our knowledge, this is the first study using TensorFlow to identify triatomines. To take advantage of deep learning algorithms to improve identification rates at the species level, we would ideally have access to many more images for each species. We are working along several lines to build such photographic resources, but clearly small sample sizes will be a continuing challenge in this work. A second major challenge, a consequence of the first, was the model validation method. We would have been happier with our results if we had enough images for each species to follow $k$-fold cross validation approaches (Kohavi 1995), instead of leave-one-out approaches to evaluate our model, as the former offer greater independence between calibration data and evaluation datasets.

Photo quality could constitute a limitation in future analysis. These problems could derive from characteristics of the photographed specimens, such as how dirty it is, the mounting orientation of the specimen, or whether or not the specimen has a pin through its thorax. Other variables that must be considered before full implementation, such as in a "citizen scientist" setting, with photographs captured with ordinary mobile phone cameras, could be evenness of lighting, distance between camera and insect, and how well focused is the image. Future experiments will evaluate the degree to which these factors compromise identification ability, to verify how robust and reliable our automated identifications can be under different circumstances.

\section{Problem species}

Although we were impressed by the improved correct identification rates that we obtained, several "problem species" remained, which need to be discussed in more detail. To identify "problem species", we considered a threshold for each dataset relative to overall country-specific identification 
rates. For Mexican species, we chose $70.0 \%$ as the threshold, which resulted in having two problem species, T. mazzottii and T. phyllosoma, with $63.6 \%$ and $69.0 \%$ overall identification rates, respectively. Total sample size for T. mazzottii was 22 images, of which 8 were misidentified, and for T. phyllosoma of 58 images, 18 were misidentified. Out of 8 incorrectly identified samples for T. mazzottii, 2 were identified as T. longipennis Usinger, and 6 as T. phyllosoma. For the 18 misidentified records of T. phyllosoma, 2 were identified as T. pallidipennis Stål, 8 as T. mazzottii, and 8 as T. longipennis. The difficulty of TensorFlow to identify these species may be related to the morphological similarity between them, as they all belong to the phyllosoma complex (Lent and Wygodzinsky 1979). However, neither morphological identity nor more recent evolution of the dimidiata complex haplogroups affected the ability to distinguish among them. Future experiments with more images should improve correct identification rates.

For Brazilian triatomine species, we selected a $75.0 \%$ correct identification threshold, below which five species had lower identification accuracy of 70-74\%: R. milesi, T. delpontei, T. guazu Lent \& Wygodzinsky, T. platensis Neiva, and T. williami Galvão, Souza \& Lima. All 7 incorrectly identified images of T. delpontei were identified as T. platensis; other problem species were confused with 2-4 other species. Again, TensorFlow limitation to distinguish between $T$. delpontei and $T$. platensis may be related to morphological similarities (Monteiro et al. 2018).

\section{Future perspectives}

In the future, we anticipate deployment of these systems more broadly in terms of both user communities and species under study. The ability to use raw images makes the process of collecting images from different sources (e.g., public health service personnel, citizen scientists) feasible, while maintaining the high accuracy of identification. Our next steps will make this technology available for 
photographs captured with ordinary mobile phone cameras, and thus broadly accessible to all via a mobile-phone application. The system will (1) permit distinguishing triatomines from non-triatomines, and (2) extend triatomine taxonomic coverage to the whole of the Americas. Extension of such approaches to other medically important arthropod groups is eminently feasible, and could be implemented with relatively little effort, at least in terms of computation. Taxa such as ticks, in particular, are significant vectors of many human and livestock pathogens (Parola et al. 2005), and could be the most suitable candidates for immediate future studies as they are larger, relatively two-dimensional and can be characterized via a single dorsal-view image. Extending the methodology to mosquitoes and other dipterans may not be too far off (Giordani et al. 2017).

\section{Acknowledgments}

We thank the Office of the Provost, of the University of Kansas, and the Consejo Nacional de Ciencia y Tecnologia (Mexico; CONACyT \#261006 to JMRW) for their support of this project. We also thank Rick Evanhoe and Riley Epperson for technical support.

\section{Author Contributions}

The entire author team conceived and designed the experiments, and reviewed drafts of the paper. Ali Khalighifar performed the experiments (with help from Ed Komp), analyzed the data, wrote the paper, and prepared figures and/or tables. 


\section{References Cited}

Abadi, M., A. Agarwal, P. Barham, E. Brevdo, Z. Chen, C. Citro, G. S. Corrado, A. Davis, J. Dean, and M. Devin. 2016. Tensorflow: Large-scale machine learning on heterogeneous distributed systems. arXiv preprint arXiv:1603.04467.

Coura, J. R., and P. A. Viñas. 2010. Chagas disease: A new worldwide challenge. Nature 465: S6-S7.

Dayhoff, J. E., and J. M. DeLeo. 2001. Artificial neural networks. Cancer 91: 1615-1635.

de Carvalho, M. R., F. A. Bockmann, D. S. Amorim, C. R. F. Brandão, M. de Vivo, J. L. de Figueiredo, H. A. Britski, M. C. de Pinna, N. A. Menezes, and F. P. Marques. 2007. Taxonomic impediment or impediment to taxonomy? A commentary on systematics and the cybertaxonomic-automation paradigm. Evol. Biol. 34: 140-143.

Drew, L. W. 2011. Are we losing the science of taxonomy? As need grows, numbers and training are failing to keep up. BioScience 61: 942-946.

Fisher, R. A. 1936. The use of multiple measurements in taxonomic problems. Ann. Hum. Genet. 7: 179188.

Giordani, B., A. Andrade, E. Galati, and R. Gurgel-Gonçalves. 2017. The role of wing geometric morphometrics in the identification of sandflies within the subgenus Lutzomyia. Med. Vet. Entomol. 31: 373-380.

Gurgel-Gonçalves, R., C. Galvão, J. Costa, and A. T. Peterson. 2012. Geographic distribution of Chagas disease vectors in Brazil based on ecological niche modeling. J. Trop. Med. 2012: 705326.

Gurgel-Gonçalves, R., E. Komp, L. P. Campbell, A. Khalighifar, J. Mellenbruch, V. J. Mendonça, H. L. Owens, K. de la Cruz Felix, A. T. Peterson, and J. M. Ramsey. 2017. Automated identification of insect vectors of Chagas disease in Brazil and Mexico: The Virtual Vector Lab. PeerJ 5: e3040.

Kohavi, R. 1995. A study of cross-validation and bootstrap for accuracy estimation and model selection, pp. 1137-1145, IJCAI, Montreal, Canada. 
Lent, H., and P. Wygodzinsky. 1979. Revision of the Triatominae (Hemiptera, Reduviidae), and their significance as vectors of Chagas' disease. Bull. Am. Mus. Nat. Hist. 163: 123-520.

Martins-Melo, F. R., C. H. Alencar, A. N. Ramos, and J. Heukelbach. 2012. Epidemiology of mortality related to Chagas disease in Brazil, 1999-2007. PLoS Negl. Trop. Dis. 6: e1508.

Monteiro, F. A., C. Weirauch, M. Felix, C. Lazoski, and F. Abad-Franch. 2018. Evolution, systematics, and biogeography of the Triatominae, vectors of Chagas disease. Adv. Parasitol. 99: 265-344.

Mukundarajan, H., F. J. H. Hol, E. A. Castillo, C. Newby, and M. Prakash. 2017. Using mobile phones as acoustic sensors for high-throughput mosquito surveillance. eLife 6: e27854.

Nauen, R. 2007. Insecticide resistance in disease vectors of public health importance. Pest Manag. Sci. 63: 628-633.

Parola, P., C. D. Paddock, and D. Raoult. 2005. Tick-borne rickettsioses around the world: Emerging diseases challenging old concepts. Clin. Microbiol. Rev. 18: 719-756.

Rampasek, L., and A. Goldenberg. 2016. TensorFlow: Biology's gateway to deep learning? Cell Syst. 2: 12-14.

Ramsey, J. M., M. Elizondo-Cano, G. Sanchez-González, A. Peña-Nieves, and A. Figueroa-Lara. 2014. Opportunity cost for early treatment of Chagas disease in Mexico. PLoS Negl. Trop. Dis. 8: e2776.

Ramsey, J. M., A. T. Peterson, O. Carmona-Castro, D. A. Moo-Llanes, Y. Nakazawa, M. Butrick, E. TunKu, K. d. la Cruz-Félix, and C. N. Ibarra-Cerdeña. 2015. Atlas of Mexican Triatominae (Reduviidae: Hemiptera) and vector transmission of Chagas disease. Mem. Inst. Oswaldo Cruz 110: 339-352.

Schmidhuber, J. 2015. Deep learning in neural networks: An overview. Neural Netw. 61: 85-117.

Schofield, C. J., J. Jannin, and R. Salvatella. 2006. The future of Chagas disease control. Trends Parasitol. 22: 583-588. 
Sinkins, S. P., and F. Gould. 2006. Gene drive systems for insect disease vectors. Nat. Rev. Genet. 7: 427435. 
Appendix 1. Summary of species analyzed, sample size in terms of numbers of photographs, and successful identification rates (\%), in our previous analyses (Gurgel-Gonçalves et al. 2017) versus using deep learning techniques, for the 12 Mexican species in this study. Hg1 - Hg3 represent three distinct haplogroups of Triatoma dimidiata complex.

\begin{tabular}{|c|c|c|c|}
\hline Species & Sample size & $\begin{array}{c}\text { Statistical } \\
\text { classifiers } \\
\text { success rate } \\
(\%)\end{array}$ & $\begin{array}{c}\text { Deep } \\
\text { learning } \\
\text { success rate } \\
(\%)\end{array}$ \\
\hline Panstrongylus rufotuberculatus (Champion, 1899) & 13 & 100.0 & 100.0 \\
\hline Triatoma barberi Usinger, 1939 & 30 & 72.4 & 80.0 \\
\hline Triatoma dimidiata (Latreille, 1811) Hg1 & 44 & 70.5 & 84.1 \\
\hline Triatoma dimidiata (Latreille, 1811) Hg2 & 30 & 76.7 & 86.7 \\
\hline Triatoma dimidiata (Latreille, 1811) Hg3 & 40 & 82.5 & 87.5 \\
\hline Triatoma gerstaeckeri (Stål, 1859) & 12 & 83.3 & 83.3 \\
\hline Triatoma longipennis Usinger, 1939 & 52 & 72.5 & 76.9 \\
\hline Triatoma mazzottii Usinger, 1941 & 22 & 77.3 & 63.6 \\
\hline Triatoma mexicana (Herrich-Schaeffer, 1848) & 45 & 80.0 & 97.8 \\
\hline Triatoma nitida Usinger, 1939 & 15 & 46.7 & 73.3 \\
\hline Triatoma pallidipennis Stål, 1872 & 44 & 90.7 & 93.2 \\
\hline Triatoma phyllosoma (Burmeister, 1835) & 58 & 46.6 & 69.0 \\
\hline Total sample size and average success rates & 405 & 80.3 & 83.0 \\
\hline
\end{tabular}


Appendix 2. Summary of species analyzed, sample size in terms of numbers of photographs, and successful identification rates (\%), in our previous analyses (Gurgel-Gonçalves et al. 2017) versus using deep learning techniques, for the 38 Brazilian species assessed in this study.

\begin{tabular}{|c|c|c|c|}
\hline Species & Sample size & $\begin{array}{c}\text { Statistical } \\
\text { classifiers } \\
\text { success rate } \\
(\%)\end{array}$ & $\begin{array}{c}\text { Deep } \\
\text { learning } \\
\text { success rate } \\
\text { (\%) }\end{array}$ \\
\hline Cavernicola lenti Barrett \& Arias, 1985 & 32 & 93.3 & 100.0 \\
\hline Panstrongylus diasi Pinto \& Lent, 1946 & 30 & 96.7 & 100.0 \\
\hline Panstrongylus geniculatus (Latreille, 1811) & 45 & 93.3 & 100.0 \\
\hline Panstrongylus lignarius (Walker, 1873) & 30 & 85.7 & 93.3 \\
\hline Panstrongylus lutzi Neiva \& Pinto, 1923 & 35 & 88.2 & 88.2 \\
\hline Panstrongylus megistus Burmeister, 1835 & 85 & 91.7 & 96.5 \\
\hline Psammolestes tertius Lent \& Jurberg, 1965 & 36 & 100.0 & 97.2 \\
\hline Rhodnius brethesi Matta, 1919 & 28 & 96.4 & 96.4 \\
\hline Rhodnius domesticus Neiva \& Pinto, 1923 & 30 & 96.3 & 96.7 \\
\hline Rhodnius milesi Carcavallo, Rocha, Galvão \& Jurberg, 2001 & 43 & 89.2 & 74.4 \\
\hline Rhodnius montenegrensis Rosa et al. 2012 & 43 & 84.6 & 88.1 \\
\hline Rhodnius nasutus Stål, 1859 & 80 & 82.2 & 88.8 \\
\hline Rhodnius neglectus Lent, 1954 & 67 & 83.3 & 90.9 \\
\hline Rhodnius pictipes Stål, 1872 & 43 & 95.3 & 97.7 \\
\hline Triatoma arthurneivai Lent \& Martins, 1940 & 32 & 78.1 & 84.4 \\
\hline Triatoma baratai Carcavallo \& Jurberg, 2000 & 29 & 82.8 & 89.7 \\
\hline Triatoma brasiliensis Neiva, 1911 & 64 & 76.6 & 92.2 \\
\hline Triatoma carcavalloi Jurberg, Rocha \& Lent, 1998 & 38 & 86.8 & 89.5 \\
\hline Triatoma circummaculata (Stål, 1859) & 22 & 85.7 & 95.5 \\
\hline Triatoma costalimai Verano \& Galvão, 1958 & 64 & 85.7 & 90.6 \\
\hline Triatoma delpontei Romana \& Abalos, 1947 & 31 & 86.7 & 74.2 \\
\hline Triatoma guazu Lent \& Wygodzinsky, 1979 & 29 & 64.3 & 72.4 \\
\hline Triatoma infestans (Klug, 1834) & 55 & 83.3 & 87.3 \\
\hline Triatoma juazeirensis Costa \& Felix, 2007 & 20 & 81.0 & 81.0 \\
\hline Triatoma lenti Sherlock \& Serafim, 1967 & 39 & 78.9 & 77.5 \\
\hline Triatoma maculata (Erichson, 1848) & 40 & 89.7 & 77.5 \\
\hline Triatoma matogrossensis Leite \& Barbosa, 1953 & 33 & 75.0 & 81.8 \\
\hline Triatoma melanica Neiva \& Lent, 1941 & 29 & 79.3 & 89.3 \\
\hline
\end{tabular}




\begin{tabular}{|c|c|c|c|}
\hline Species & Sample size & $\begin{array}{c}\text { Statistical } \\
\text { classifiers } \\
\text { success rate } \\
(\%) \\
\end{array}$ & $\begin{array}{c}\text { Deep } \\
\text { learning } \\
\text { success rate } \\
(\%) \\
\end{array}$ \\
\hline Triatoma pintodiasi Jurberg, Cunha \& Rocha, 2013 & 26 & 88.0 & 84.0 \\
\hline Triatoma platensis Neiva, 1913 & 28 & 74.1 & 71.4 \\
\hline Triatoma pseudomaculata Correa \& Espínola, 1964 & 56 & 70.9 & 75.0 \\
\hline Triatoma rubrovaria (Blanchard, 1843) & 55 & 59.3 & 79.6 \\
\hline $\begin{array}{l}\text { Triatoma sherlocki Papa, Jurberg, Carcavallo, Cerqueira \& } \\
\text { Barata, } 2002\end{array}$ & 32 & 93.5 & 90.6 \\
\hline Triatoma sordida (Stål, 1859) & 96 & 81.2 & 92.7 \\
\hline Triatoma tibiamaculata (Pinto, 1926) & 41 & 92.7 & 85.4 \\
\hline $\begin{array}{l}\text { Triatoma vandae Carcavallo, Jurberg, Rocha, Galvão, Noireau } \\
\text { \& Lent, } 2002\end{array}$ & 30 & 69.0 & 79.3 \\
\hline Triatoma vitticeps (Stål, 1859) & 48 & 85.1 & 80.9 \\
\hline Triatoma williami Galvão, Souza \& Lima, 1965 & 20 & 70.6 & 70.0 \\
\hline Total sample size and average success rates & 1584 & 83.9 & 86.7 \\
\hline
\end{tabular}

